Cinémas

Revue d'études cinématographiques

Journal of Film Studies

\title{
Le film comme lieu de mémoire : En el balcón vacío et l'exil mexicain des Espagnols
}

\section{Vicente Sánchez-Biosca}

Volume 15, numéro 1, automne 2004

Entre l’Europe et les Amériques

URI : https://id.erudit.org/iderudit/011659ar

DOI : https://doi.org/10.7202/011659ar

Aller au sommaire du numéro

Éditeur(s)

Cinémas

ISSN

1181-6945 (imprimé)

1705-6500 (numérique)

Découvrir la revue

Citer cet article

Sánchez-Biosca, V. (2004). Le film comme lieu de mémoire : En el balcón vacío et l'exil mexicain des Espagnols. Cinémas, 15(1), 65-79.

https://doi.org/10.7202/011659ar
Résumé de l'article

L'expérience de l'exil en tant que dépossession a d'habitude été transmise de façon orale. Moins fréquente est la mise en images de cette expérience ou, autrement dit, l'utilisation du film comme support de la mémoire de l'exilé. Tel est le cas du film En el balcón vacío (1961), scénarisé par María Luisa Elío et réalisé par Jomí García Ascot. Offrant un support fictionnel aux souvenirs traumatiques de la guerre civile espagnole et de l'exil mexicain de la scénariste, García Ascot réalisa le seul film espagnol entièrement consacré à la mémoire d'une certaine Europe au sein des Amériques. 


\section{Le film comme lieu de mémoire: En el balcón vacio et l'exil mexicain des Espagnols}

\section{Vicente Sánchez-Biosca}

\section{RÉSUMÉ}

L'expérience de l'exil en tant que dépossession a d'habitude été transmise de façon orale. Moins fréquente est la mise en images de cette expérience ou, autrement dit, l'utilisation du film comme support de la mémoire de l'exilé. Tel est le cas du film En el balcón vacio (1961), scénarisé par María Luisa Elío et réalisé par Jomí García Ascot. Offrant un support fictionnel aux souvenirs traumatiques de la guerre civile espagnole et de l'exil mexicain de la scénariste, García Ascot réalisa le seul film espagnol entièrement consacré à la mémoire d'une certaine Europe au sein des Amériques.

\section{ABSTRACT}

The experience of exile as dispossession is usually passed on orally. The use of images to describe this experience, or the use of film as a support to the exile's memory, is less frequent. En el balcón vacío (1961), written by María Luisa Elío and directed by Jomí García Ascot, is such an example. Using fictional elements to support Elío's own traumatic memories of the Spanish civil war and the Mexican exile, García Ascot realized the only Spanish film entirely dedicated to the persistent memory of a specific Europe within the Americas. 
La verdadera nostalgia, la más honda, no tiene que ver con el pasado, sino con el futuro. Yo siento con frecuencia la nostalgia del futuro, quiero decir, nostalgia de aquellos dias de fiesta, cuando todo merodeaba por delante y el futuro aún estaba en su sitio.

Luis García Montero

\section{Les autres Amériques}

«Faire les Amériques» est une expression utilisée de longue date dans la tradition espagnole; elle est synonyme de réussite, surtout économique, dans les pays du Nouveau Monde. L'Amérique dont il est question ici est celle que les Européens du Sud voyaient comme un espace qui, sans être totalement étranger, était encore vierge, libre et propice à l'ascension sociale. Héritière des colonies d'autrefois, cette Amérique partage avec elles la langue, la religion et la culture, même si cette dernière devait négocier avec les cultures autochtones. C'est dans ce lieu physique et imaginaire des Amériques que maints gachupines s'installèrent au fil des siècles. Ils suscitèrent de la part des Américains des jugements tantôt critiques, tantôt amicaux, ou les deux à la fois. Il n'est pas étonnant que ces exilés espagnols aient eu de temps à autre le mal du pays et, peut-être aussi, qu'ils aient songé à un retour vers leur patrie d'origine. Pour nous en tenir aux temps récents, soulignons qu'au cours des cinquante années qui précédèrent la proclamation de la II $^{e}$ République espagnole (1931), environ quatre millions d'Espagnols ont émigré en Amérique latine (Sánchez Albornoz 1991, p. 17). Cependant, les raisons de cette vague d'émigration (principalement, l'espoir d'une réussite économique et sociale) n'ont rien à voir avec celles qui motivèrent une autre vague qui se produisit après le traumatisme de la guerre civile espagnole et l'échec de la II République. Dès 1939, l'exode des républicains espagnols n'est plus motivé par l'espoir de réussite, en quelque domaine que ce soit, mais par la simple nécessité de survivre.

Nombreuses sont les différences entre les émigrés d'autrefois et les réfugiés républicains, mais nous n'en retiendrons que deux: 
les réfugiés républicains sont des défenseurs ardents de la démocratie, ce que n'étaient pas nécessairement leurs prédécesseurs, et ils ne sont généralement pas de même provenance sociale (notamment, leur niveau d'éducation est en moyenne plus élevé, mais pas autant que certains ont pu le laisser entendre).

Parmi les exilés, les uns se dirigèrent vers la France ou d'autres pays d'Europe de l'Ouest (Belgique, Angleterre...), les autres vers l'Union soviétique. Les uns comme les autres (exceptés ceux qui se rendirent en Angleterre) eurent bientôt à vivre une seconde expérience traumatique, sous la forme d'une nouvelle guerre et d'une deuxième persécution (le cas de l'écrivain Jorge Semprun qui, comme des milliers d'Espagnols, fut déporté à Mauthausen, en offre un exemple bien connu). Les plus heureux furent ceux qui s'acheminèrent vers l'Amérique latine, soit vers le Mexique, soit vers la République dominicaine, soit vers l'Argentine ou d'autres pays. Grâce à la proportion non négligeable d'intellectuels qu'ils comptaient dans leurs rangs, à la langue commune qu'ils parlaient et au calme relatif dans lequel se déroula leur exil, ces réfugiés furent en mesure d'élaborer petit à petit une sorte de "roman national du retour", sous forme de récit mélancolique. C'est ainsi qu'ils s'efforcèrent de rebâtir là-bas une Espagne à petite échelle, autant du point de vue politique que du point de vue professionnel, éducationnel et quotidien. L'espoir d'un retour imminent vers la terre natale après l'intervention militaire des forces alliées en Espagne pour renverser la dictature franquiste céda peu à peu le pas à une lente prise de conscience du temps, grâce à laquelle la donnée biographique rejoignit l'histoire collective. C'est ainsi que l'exil devint un état de conscience.

Le Mexique fut un lieu d'accueil spécialement agréable pour les réfugiés. D’une part, le président Lázaro Cárdenas avait déjà contribué à la cause de la République pendant la guerre civile, et, après la fin de celle-ci, il donna son accord à l'immigration de quelques 20000 réfugiés, à la condition que le SERE (Servicio de Evacuación de Republicanos Españoles) prît en charge leur traversée. De surcroît, les réfugiés purent bénéficier d'un accueil très favorable: l'esprit révolutionnaire du PRI (Partido Revolucionario Institucional) se trouvait en phase avec 
celui des libéraux et des gauchistes, qui formaient le plus gros des rangs de cet exil espagnol, ce qui n'était pas le cas avec les émigrés traditionnels - les gachupines - dont la tendance politique était de droite (nombreux sont ceux qui donnèrent leur soutien à Franco ${ }^{1}$ ). Grâce à ces conditions favorables, les réfugiés purent revivre l'expérience républicaine brutalement interrompue, en mettant sur pied diverses institutions (écoles, centres culturels, collèges), ainsi que des maisons d'édition, des revues, etc. De cette effervescence sont nées plusieurs institutions, qui devinrent avec le temps des lieux de mémoire (dans le sens que Pierre Nora, 1997, a donné à ce terme), dédiés à une Espagne perdue à jamais: le Centro Republicano Español; l'Ateneo Español de México ; la Casa de España (1938), devenue le Colegio de México en 1940; de nombreux collèges (l'Instituto Luis Vives, le Colegio Madrid, l'Academia HispanoMexicana). Notons aussi la fondation, en août 1945, du gouvernement républicain en exil, qui établit son premier siège à Mexico. Présidé par José Giral, il fut reconnu par le gouvernement mexicain comme le représentant légitime de l'Espagne, le gouvernement mexicain rejetant ainsi toute relation diplomatique avec l'État du général Franco, qui ne fut jamais reconnu par le Mexique ${ }^{2}$.

Cet ensemble de circonstances provoqua chez les exilés une impression fort paradoxale d'adaptation, radicalement différente de celle que reçurent les immigrants précédents (Pla Brugat 2001). Le philosophe José Gaos (1966, p. 177) décrit la situation en ces termes: «[...] le manque d'impression d'exil chez les réfugiés adaptés; la présence en eux d'une impression de s'être transportés d'une terre espagnole à une autre, qu'il faudrait plutôt appeler, par conséquent, impression de trans-rapatriement ${ }^{3}$." Cependant, l'impression de vivre dans une petite Espagne finit par brouiller les sentiments au point d'être cause d'illusion, d'autant plus que le retour espéré était sans cesse reporté à plus tard et s'avéra bientôt impossible.

\section{Le cinéma comme absence}

S'il est vrai que les réfugiés espagnols ne cessèrent de construire à l'intérieur du Mexique des lieux en lesquels ils 
reconnaissaient leur patrie (ou, pour mieux dire, une Espagne républicaine désormais imaginaire), force est de constater que le support filmique ne contribua guère à créer cet esprit d'exil, ni au Mexique ni ailleurs. Cela tient probablement aux caractéristiques industrielles du cinéma, aux ressources financières qu'exige la production cinématographique et à son mode de fonctionnement collectif, car de nombreux exilés espagnols qui travaillaient dans le domaine du cinéma parvinrent avec une relative facilité à s'établir dans d'autres circuits de production, que ce soit en Argentine, au Mexique ou ailleurs, avec ou sans succès, mais sans éprouver - explicitement du moins - de nostalgie ${ }^{4}$. Un cas tout à fait paradigmatique est celui de Luis Buñuel: impossible de ne pas reconnaître en lui les traits des républicains (militance politique et esthétique héritière des avant-gardes, refus du franquisme et adoption ultérieure, dans un geste symbolique, de la nationalité mexicaine ${ }^{5}$ ). Par ailleurs, on ne manquera pas de repérer dans son œuvre cinématographique mexicaine un imaginaire de tradition hispanique (omniprésence de rites religieux, types et caractères des personnages, clin d'œils musicaux, fragments de conversations faisant allusion à l'Espagne, éléments folkloriques...). Néanmoins, la mémoire de l'Espagne en tant qu'objet perdu ne joue pratiquement aucun rôle dans ses films. Le cas n'est pas exceptionnel chez les exilés, mais il est plus éloquent chez Buñuel, étant donné sa réputation internationale.

Un seul film résume vraiment l'expérience intense de l'exil espagnol, tout en se portant garant de la mémoire de l'Espagne abandonnée; il s'agit d'un film dont le sujet est la double fracture, dans la vie d'un enfant, provoquée par la guerre civile et par l'exil. Basé sur un texte de María Luisa Elío, En el balcón vacío est une reconstitution imaginaire de la fuite et du retour en Espagne. Réalisé la même année que Viridiana, coproduction hispanomexicaine, En el balcón vacío eut très tôt un impact social dépassant largement celui habituellement produit par une œuvre d'art: les qualités particulières de sa production, de son sujet, de son scénario et de sa distribution firent que ce film devint un lieu de mémoire pour la communauté des exilés. Il semble donc légitime de se pencher sur sa cartographie imaginaire. 


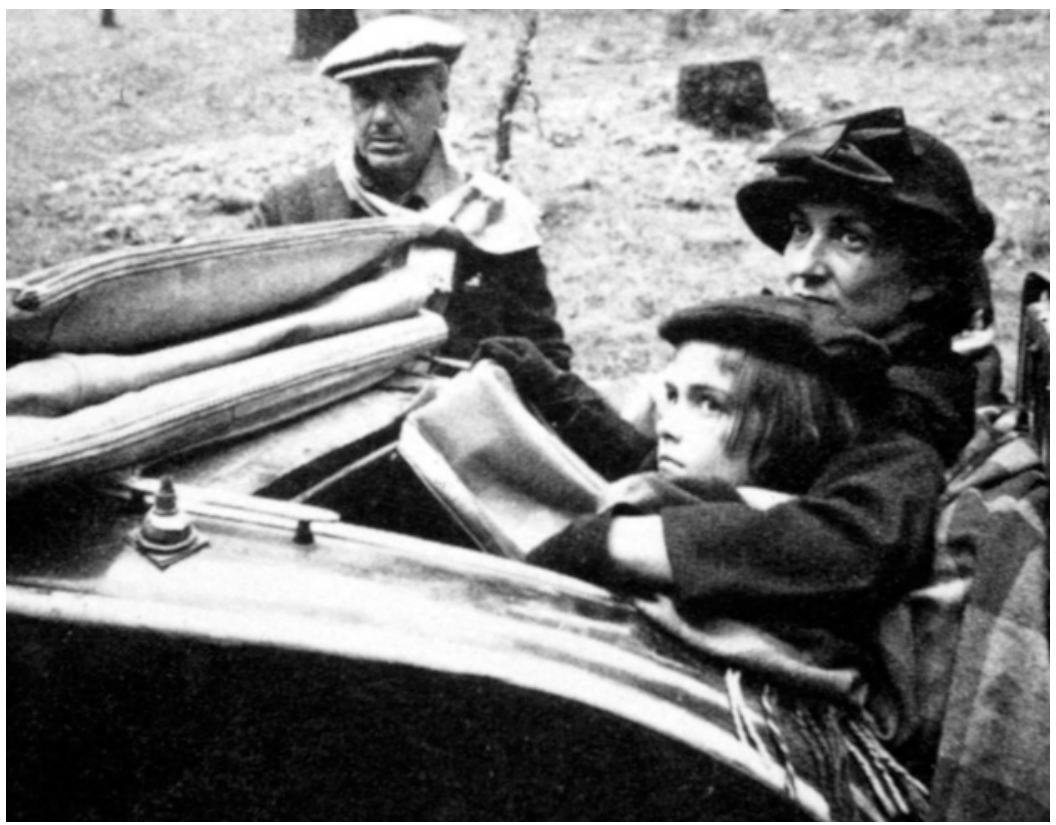

L’exil vers la France (En el balcón vacio, Jomí García Ascot)

(C) Collection personnelle de la famille

Film culte, lieu de mémoire

Lors d'entretiens, María Luisa Elío a raconté comment, un jour, des souvenirs liés à son exil resurgirent en elle, déclenchant ainsi le processus d'écriture de En el balcón vacío. Lorsque, en 1959, son époux Jomí García Ascot fut appelé à La Havane par Alfredo Guevara, directeur de l'ICAIC révolutionnaire, des images de combattants dévalant les montagnes vers la capitale lui revinrent en mémoire, et ces images éveillèrent d'autres souvenirs: de la petite enfance, de la persécution et de l'exil. Mais quel que fût l'esprit individuel et personnel dans lequel s'enracinaient les souvenirs d'Elío et son écriture, le film qui en a résulté devint aussitôt un lieu de mémoire partagé par une communauté d'exilés installés au Mexique. Le processus de création du film le prouve sans l'ombre d'un doute.

En el balcón vacio est une ouvre collective familiale, une production domestique. Le film fut tourné en quarante jours; plus précisément: quarante dimanches - le dimanche étant un jour férié où tous les amis pouvaient se réunir —, entre 1961 et 
1962. L'appareil servant aux prises de vues ne pouvait qu'être un appareil amateur: une ancienne caméra Pathé-Webbo à corde de $16 \mathrm{~mm}$. Quant aux sources de financement, elles étaient réduites à l'apport des amis (4000 dollars), auquel s'ajoutèrent les fruits de la vente de trois tableaux de peintres exilés: Vicente Rojo, Juan Soriano et Souza ${ }^{6}$.

Concernant les lieux de tournage, étant donné le sujet du film, la plupart devaient permettre la reconstitution de décors espagnols. On choisit donc de tourner dans des décors naturels, plus particulièrement ceux qu'offraient les bâtiments d'architecture espagnole de la "petite Espagne»: le Sanatorio Español, le Panteón Español, le Colegio Madrid, l'Ateneo Español, le Parque Lira, le Colegio de España. Dans ce choix, il n'est pas seulement question de lieux physiques de tournage, mais d'un exercice pragmatique d'une importance capitale: récupérer ce qui avait été perdu à l'aide d'un «bricolage», en s'appropriant une réalité mexicaine préalablement "espagnolisée " par l'intervention esthétique, politique et sociale des exilés eux-mêmes. C'est ainsi que la mémoire pouvait resurgir. De tels lieux deviennent, pour les républicains, des évocations in absentia de lieux égarés, de lieux désormais inaccessibles. Cette résistance opiniâtre du groupe à l'oubli se veut un acte de témoignage destiné aux enfants. María Luisa Elío raconte le premier jour de tournage dans le Désert des lions ${ }^{7}:$ «[...] tous les exilés étaient habillés avec des vêtements de 1936; il se fit un grand silence, comme s'il s'agissait d'une vraie hallucination, d'une émergence du passé. Lorsque la caméra cessa de filmer, des larmes coulaient sur les joues de tous les participants» (Alonso 1999, p. 145).

Notons enfin que les protagonistes du film (acteurs et membres de l'équipe technique) appartiennent tous, à quelques exceptions près, à la communauté des exilés. Ils sont soit de la deuxième génération (ceux qui quittèrent l'Espagne, enfants, et qui n'avaient pas participé à la guerre), soit de la troisième génération (ceux qui sont nés pendant l'exil, comme la fillette qui interprète Gabriela, Nuri Pereña, qui fut doublée étant donné son accent mexicain). Ces particularités font que la nostalgie de la patrie se mêle à la nostalgie de l'enfance. Le récit retrace, à quelques détails près, le parcours de María Luisa Elío: 


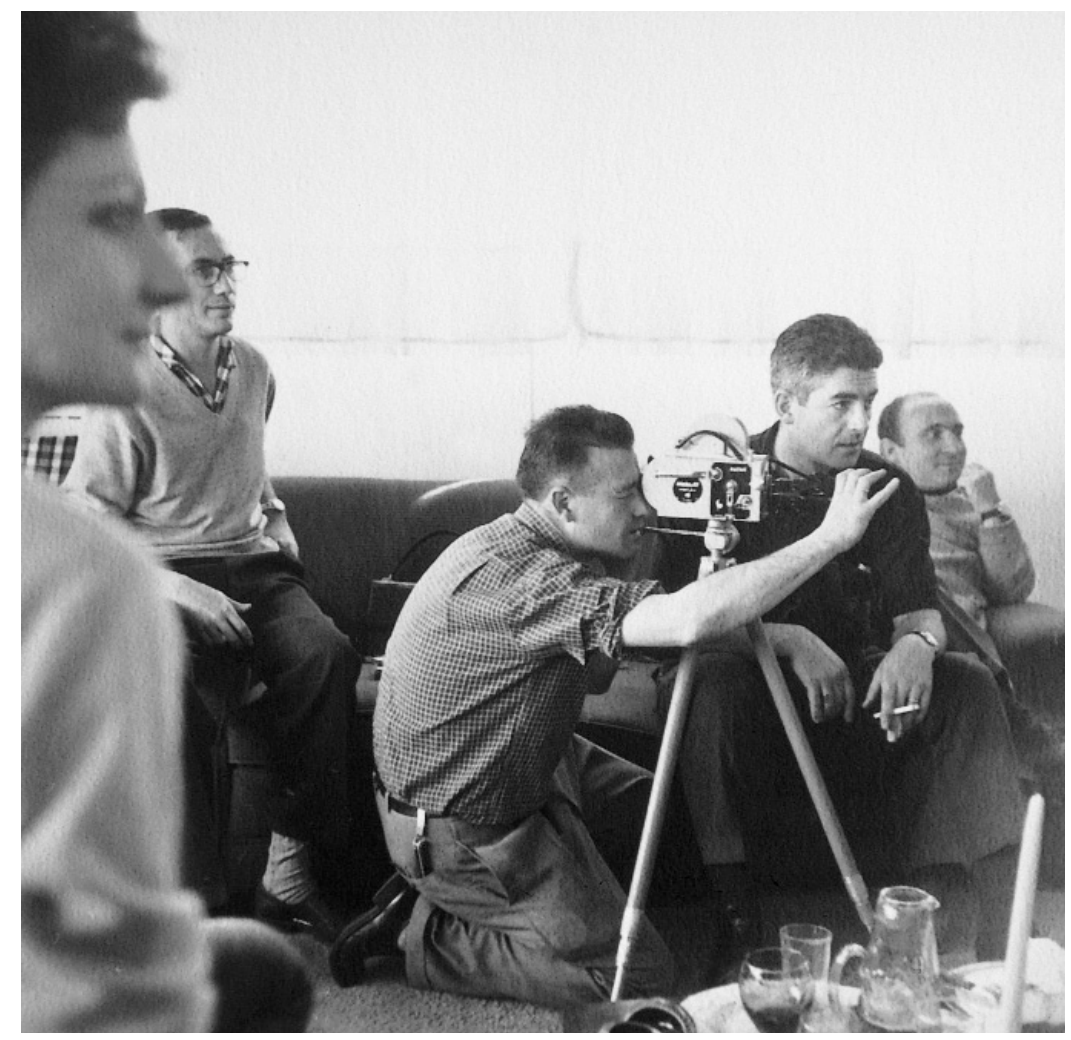

Pendant le tournage du film En el balcón vacío (Jomí García Ascot) (C) Collection personnelle de la famille

seule la séquence du retour à la ville natale fut entièrement inventée. Son époux, José María García Ascot (né en 1928), fut également exilé en France et ensuite au Mexique; l'assistant et coscénariste Emilio García Riera est l'un des plus célèbres historiens du cinéma mexicain. Les titres ont été conçus par le peintre Vicente Rojo et la photographie est due à José María Torre (photographe publicitaire et époux de Conchita Genovés, qui joue la mère dans le film), assisté de José Redondo ${ }^{8}$.

Malgré les quelques prix qui lui furent décernés (Prix international de la critique à Locarno, en Suisse; Jano d'Or au Rassegna Latinoamericana de Sestri-Levante en 1963), la commercialisation du film s'avéra impossible et sa diffusion fut limitée à des cercles restreints ou familiaux. Le film fut projeté dans des domiciles privés à l'occasion de réunions de la communauté des 
exilés et dans certains ciné-clubs. Cela lui valut de devenir un film culte sur l'exil mexicain et, au bout du compte, sur l'exil républicain espagnol en général (Alonso 1999, p. 147).

Tout ce que l'on vient d'exposer démontre que ce film est un produit quasi familial, devenu le lieu de reconnaissance d'une communauté et ensuite un vrai lieu de mémoire, c'est-à-dire un lieu où s'ancrent des témoignages de l'exil, et ce à un moment où la mémoire de l'exil tend à se désagréger.

Malgré une certaine parenté avec l'esthétique intimiste adoptée par maints réalisateurs attachés au thème de la mémoire, avant d'être expérimental, le film de García Ascot est surtout moderne. Par exemple, les longs plans «documentaires" de Mexico doivent être mis au compte du réalisme, propre à l'esprit du nouveau cinéma latino-américain du début des années 1960. De plus, l'intimisme - l'expérience unique et individuelle - du sujet (le journal filmique) cède ici le pas à une entreprise collective, d'où résulte un film collectif, ne seraitce que de second ordre. Or, quelle est la forme plastique et filmique qu'adopte l'idée d'exil?

\section{Une forme plastique pour la désolation}

En el balcón vacio raconte l'histoire d'un voyage, celui d'une femme, Gabriela, dont la voix off conduit le récit. À huit ans, Gabriela est témoin des débuts de la guerre civile espagnole, qui plonge sa famille jusque-là heureuse dans le désarroi. La famille - dont les membres sont de gauche - est traquée, Pamplona, la ville qu'elle habite, étant tombée aux mains des insurgés fascistes. Le père qui, selon les indices que donne le film, est un activiste, est forcé de se cacher et de disparaître. Un jour, à travers la fenêtre de sa chambre, Gabriela voit un homme terrifié se cacher des policiers qui le poursuivent, puis une femme le dénoncer avec un cri qui épouvante l'enfant. Ce souvenir demeurera fixé dans sa mémoire de manière indélébile, comme une déclaration de guerre. La famille est contrainte d'entreprendre un périple qui mènera la mère et les deux filles à la zone contrôlée par le gouvernement de la République. Ensuite, après la chute du front républicain, la famille s'exile en France et, enfin, au Mexique. Les vues de la ville de Mexico ouvrent une 
séquence condensant vingt ans de la vie de Gabriela, au bout desquels elle se trouve seule, sans famille, sa mère étant décédée entre-temps. Cela la conduit à fantasmer un voyage de retour: le film nous transporte en effet au cœur de ce qui semble être une hallucination, pendant laquelle Gabriela se rencontre elle-même enfant. Gabriela est dans la maison vide de son enfance, près d'une fenêtre grande ouverte, tandis qu'elle croit entendre la voix de son père et plonge dans un délire tragique dont le sujet est le passage du temps.

L'image de Gabriela enfant venant se heurter à une fenêtre vide - surface immense, démesurée et déserte, censée incarner symboliquement sa mémoire défaillante — est exploitée à plusieurs reprises tout au long du film. Cette image de la fenêtre vide, sorte de "forme-concept", explique d'ailleurs le titre du film. On pourrait même dire que le film en son entier n'est qu'une série de variantes spatiales et narratives sur cette forme-concept.

La séquence d'ouverture nous situe dans la maison familiale, alors que le signifiant "guerre" fait irruption dans le monde innocent de Gabriela. Ce signifiant brise violemment l'atmosphère idyllique du foyer, où père, mère et fille se rassemblent autour d'une table, tandis que résonne une musique de Bach. Sous le regard surpris de Gabriela, un fugitif descend alors du toit de la maison voisine et se cache dans l'embrasure de la fenêtre. La terreur se devine sur le visage de l'homme alors que Gabriela le regarde avec une sympathie croissante. Une étrange communion s'établit entre le fugitif et l'enfant, communion que le film exprime à travers un champ-contrechamp qui contredit la distance physique séparant les personnages en soulignant leur proximité affective. Un plan ayant une large profondeur de champ capte ensuite la scène à travers la fenêtre gigantesque où l'enfant, à l'avant-plan, plonge comme dans un abîme. C'est également par cette fenêtre ouverte sur le monde que le signifiant "guerre», par l'entremise du cri dénonciateur d'une femme qui provoque l'arrestation du fugitif, envahira l'univers de Gabriela. Ce cri sauvage brise l'innocence du monde de Gabriela qui, terrifiée, s'exclame: "Maman, la guerre est arrivée!» Gabriela quitte le cadre et la caméra filme alors un immense espace vacant, le décor de la scène dramatique désormais vide, 
métaphore de l'apprentissage traumatique que vient de faire l'enfant. Le cri dénonciateur qui, par métonymie, annonçait la guerre, fait place à une image de désolation qui désigne la mort. C’est ainsi que la première expérience de la mort est représentée par le vide - et ce vide réapparaîtra à plusieurs reprises dans le film, comme une rime tragique, sous diverses formes.

La situation de la famille étant devenue critique, et le père ayant fui la maison, Gabriela assiste à une autre scène chargée d'émotions. Légèrement en retrait par rapport à eux, elle voit quelques enfants qui lancent des pierres vers une prison improvisée où un homme immobile - un républicain, "un rouge" selon le discours franquiste - regarde derrière les grilles. Le prisonnier discerne la présence de Gabriela, silencieuse au milieu des cris des autres enfants. La composition du cadre rappelle clairement le plan de la fenêtre analysé plus haut, si bien que les grilles de la prison, qui symbolisent avec force la privation de liberté, paraissent avoir été fixées à cette fenêtre, comme si c'était elle qu'on avait barricadée. Le prisonnier sourit à Gabriela et, malgré la distance qui les sépare, le montage les

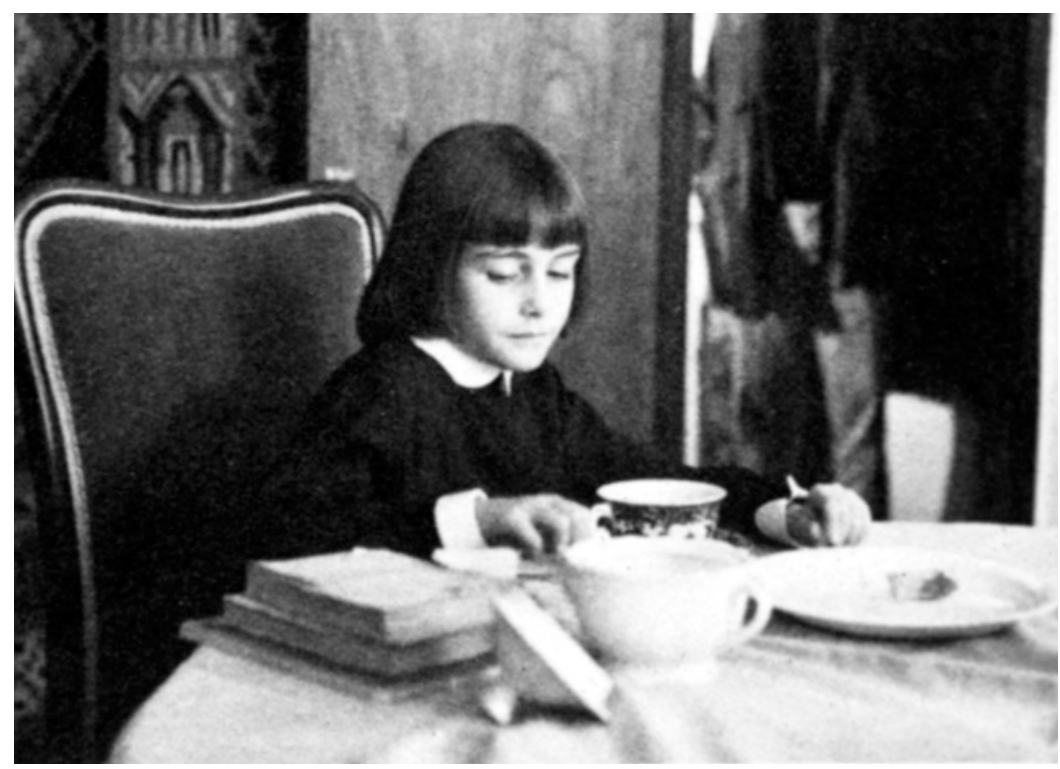

Le temps de l'enfance et de la guerre (En el balcón vacío, Jomí García Ascot) (C) Collection personnelle de la famille 
fait se rejoindre grâce à un champ-contrechamp qui nous libère des contraintes de l'espace et nous conduit dans un monde affectif intérieur. Les sourires compatissants que les deux personnages s'échangent construisent un pont entre eux, et la séquence met ainsi à nouveau en lumière les affinités électives de Gabriela, déjà mises en évidence au cours de la séquence du fugitif?.

Cette compassion de Gabriela à l'égard du proscrit la distingue du reste des enfants et confirme sa sensibilité humaniste, plutôt que politique. Cette sensibilité est toujours mise en relation avec le personnage absent du père, dont Gabriela apprendra la mort un peu plus tard. La caméra se déplace à l'intérieur de la prison afin de montrer la silhouette du prisonnier à l'avant-plan; elle cadre ensuite l'immense grille et, au fond, toute petite, la fillette. La place reste déserte, mais un sourire reste sur les lèvres du prisonnier lorsqu'une voix impérieuse venue de l'intérieur de la prison l'appelle et que celui-ci disparaît. Le caractère tragique de l'épisode est confirmé par le destin du prisonnier. En effet, le lendemain, la fillette apprendra qu'il a été fusillé. Derrière la grille, il n'y a maintenant plus personne et, en contrechamp, de l'intérieur de la prison, on voit Gabriela. Quelque chose a changé par rapport à la première séquence: Gabriela est maintenant filmée en contrechamp, du point de vue de la victime, encadrée par cette sorte de fenêtre vide qui, auparavant, était vue de l'extérieur.

La veille de son départ pour le Mexique, Gabriela entend les nouvelles du soir, annoncées dans une langue inconnue, le français. Au milieu de cette atmosphère étrangère, quelques mesures de musique du compositeur espagnol Tomás Bretón se font entendre, provenant d'un appartement voisin. La fillette se lève et se penche à la fenêtre: un gros plan la montre avec des larmes sur les joues. "J'avais connu la nostalgie et maintenant je connaissais l'exil ", dit la voix off. Cette scène est le corollaire des deux séquences précédentes: là où avant il y avait complicité avec le fugitif ou le prisonnier, maintenant il n'y a plus aucun échange, aucune compassion; maintenant le vide intérieur, la solitude se dévoilent. Il n'est plus question de fenêtre ouverte sur un monde cruel: la perte est désormais intériorisée et l'absence a 
pris sa place. Impression de dépossession et mal du pays se révèlent des sentiments différents de la nostalgie: un état de conscience nouveau en résulte. Le vide matériel est le signifiant de cette terrible absence de trace mnésique.

La dernière séquence du film nous transporte dans un univers hallucinatoire, où se produit le retour vers le foyer. Celui-ci est désert et à peine reconnaissable. Sur les escaliers, Gabriela adulte croise à trois reprises Gabriela enfant, entre dans la maison sans meubles et retrouve la fenêtre vide grande ouverte, pendant que la voix off commente la taille adulte de son corps et discourt sur le temps. L'enfant Gabriela apparaît à la fenêtre alors que Gabriela adulte la regarde depuis l'avant-plan. Ensuite, l'adulte se penche à la fenêtre, mais la petite fille a déjà disparu. Un monologue portant sur le passage du temps s'amorce: «Et maintenant je me rends compte que retourner c'est partir " (Elío 1988, p. 17). Tandis que Gabriela cherche une marque laissée autrefois sous la fenêtre, sa conscience se brouille et elle paraît mentalement troublée. Elle croit entendre la voix de son père l'appelant pour souper et la psychose se déclenche. À l'image de la fenêtre, figure symbolique, même si traumatique, de l'entrée dans le monde, succède un gros plan "claustrophobique" de Gabriela, qui clôt le film; il n'y a pas d'autre échappatoire que la folie, pas de retour possible ${ }^{10}$. Le lieu de la fenêtre se voit maintenant envahi par un visage saisi de frayeur.

\section{Conclusion}

En somme, En el balcón vacío constitue non seulement un document sur l'exil des Espagnols républicains au Mexique, mais aussi un vrai lieu de mémoire, construit sur support filmique, ayant permis à une communauté de se reconnaître et de resserrer les liens entre ses membres au fil des années. Ce lieu de mémoire a été construit non seulement sur la fabulation d'une Espagne perdue à jamais, mais sur d'autres lieux de mémoire bâtis préalablement par les exilés espagnols (monuments, écoles, centres de recherche...). De ce point de vue et sans doute à petite échelle, En el balcón vacío joue un rôle similaire à Shoah, film de Claude Lanzmann (1985), qui s'acharna à créer des lieux de mémoire de l'extermination du peuple juif par les 


\section{nazis en Pologne, où les Juifs n'existaient plus, la Pologne devenant ainsi un lieu de reconnaissance monumental. Dès lors, l'analyse d'un tel film, de même que l'analyse d'un film comme En el balcón vacio, exige la double compétence de l'historien généraliste et de l'historien du cinéma; autrement dit, elle demande la présence conjointe de l'esthétique et de l'histoire.}

\section{Universitat de València}

\section{NOTES}

1. Par ailleurs, l'orientation indigéniste et anticolonialiste des factions révolutionnaires mexicaines aurait été en contradiction avec un soutien aux représentants de l'ancienne métropole.

2. Concernant les avatars de la formation de ce gouvernement, ainsi que les dispositions financières du gouvernement mexicain à son endroit, voir Cabeza SánchezAlbornoz 1997.

3. Lauteur joue sur les expressions espagnoles destierro et transtierro (expatrier et «transpatrier»; expatriement et "transpatriement»).

4. Il y a trente ans déjà, un premier bilan fut dressé par Román Gubern (1976), et aucun autre ouvrage ne l'a contesté jusqu'à présent. En ce qui concerne les productions mexicaines, voir le bilan établi par Juan Rodríguez (1997).

5. Par ailleurs, on peut se rappeler les films de propagande de Buñuel, que ce soit le documentaire Espagne 1936, réalisé lors de son séjour à l'ambassade d'Espagne à Paris, dans lequel il milite en faveur de la République légitime contre Franco, ou le remontage du Triomphe de la volonté de Leni Riefenstahl, réalisé plus tard à New York.

6. Voir les données qu'a réunies Alonso (1999) sur la production et la distribution du film.

7. Desierto de los leones: grande forêt près de la capitale mexicaine.

8. Ont également participé au film: José de la Colina, le poète Tomás Segovia, le publiciste Jaime Muñoz de Baena (le père), Francisca Riera, mère de Emilio García Riera (femme qui apparaît dans la gare), Carmen Meda (sœur de Gabriela à l'âge adulte)...

9. On peut par ailleurs attirer l'attention sur une autre scène illustrant un échange en champ-contrechamp, lorsqu'un policier interroge Gabriela au sujet de son père. Dans cette scène, la proximité physique de l'homme ainsi que sa voix délibérément séductrice éveillent la méfiance de la jeune fille qui garde un silence prudent, bien différent de ce silence complice dont on vient de rendre compte.

10. Le retour, pour María Luisa Elío, ne se produisit réellement qu’en 1970. Dans le texte qui sert de base au scénario et dans le scénario lui-même, il s'agit d'un fantasme de retour. Le fantasme a dû marquer profondément l'expérience réelle; c'est ce que laisse entendre le témoignage écrit de 1970 (Tiempo de llorar).

\section{RÉFÉRENCES BIBLIOGRAPHIQUES}

Alonso 1999: Charo Alonso, "Una mirada hacia lo perdido: En el balcón vacio", Archivos de la Filmoteca, n ${ }^{\circ} 33,1999$, p. 140-149. 
Alted Vigil 1999 : Alicia Alted Vigil, «En el balcón vacio o la confluencia entre escritura fílmica y escritura histórica", Archivos de la Filmoteca, n 33, 1999, p. 130139.

Aznar Soler 1997 : Manuel Aznar Soler, «Cultura y literatura del exilio republicano español de 1939 en México ", Taifa, n 4, 1997, p. 15-40.

Cabeza Sánchez-Albornoz 1997 : Sonsoles Cabeza Sánchez-Albornoz, Historia política de la Segunda República en el exilio, Madrid, Fundación Universitaria Española, 1997.

Company 1999: Juan Miguel Company, «El exilio y el Reino. Cinco notas sobre En el balcón vacion", Archivos de la Filmoteca, n³3, 1999, p. 162-167.

Elío 1988: María Luisa Elío, Tiempo de llorar, Mexico, Ediciones del Equilibrista, 1988.

Elío 1995: María Luisa Elío, "En el balcón vacío», Cuadernos de apuntes, Mexico, Consejo Nacional para la Cultura y las Artes/Ediciones del Equilibrista, 1995.

Gaos 1966: José Gaos, «La adaptación de un español a la sociedad hispanoamericana", Revista de Occidente, $\mathrm{n}^{\circ} 38,1966$, p. 168-178.

García Riera 1979: Emilio García Riera, Historia documental del cine mexicano, Mexico, Era, 1979.

Gubern 1976: Román Gubern, El cine español en el exilio, Barcelone, Seix Barral, 1976.

Naharro-Calderón 1999: José María Naharro-Calderón, "En el balcón vacío de la Memoria y la memoria de En el balcón vacio", Archivos de la Filmoteca, n³3, 1999, p. 150-161.

Nora 1992 : Pierre Nora, Les Lieux de mémoire, Paris, Gallimard, 1992.

Pla Brugat 2001 : Dolores Pla Brugat, "La presencia española en México, 19301990. Caracterización e historiografía", Migraciones \& Exilios, n² 2, 1990, p. $157-$ 188.

Rodríguez 1997: Juan Rodríguez, «La aportación del exilio republicano español al cine mexicano", Taifa, no 4, 1997, p. 197-224.

Sánchez Albornoz 1991 : Nicolás Sánchez Albornoz (dir.), El destierro español en América. Un trasvase cultural, Madrid, Siruela, 1991. 